\title{
EP-70
}

\section{Outcomes of deceased donor liver transplantation from elderly donors}

\author{
Minjae KIM, Shin HWANG*, Chul-Soo AHN, Ki-Hun KIM, Deok-Bog MOON, Tae-Yong HA, \\ Gi-Won SONG, Dong-Hwan JUNG, Gil-Chun PARK, Young-In YOON, Sung-Gyu LEE
}

Department of Surgery, Asan Medical Center, University of Ulsan College of Medicine, Seoul, Korea

Introduction: Favorable outcomes achieved after deceased donor liver transplantation (DDLT) suggest that use of elderly donors may be an effective way to expand donor pool.

Methods: This was a retrospective analysis of adult DDLT using elderly donors. It was a double-arm study that compared posttransplant outcomes to ascertain whether use of elderly donors (aged $\geq 76$ years) has adverse effects on outcome of DDLT. The elderly study group included 14 donors aged $\geq 76$ years and the elderly control group comprised 39 donors aged 66-75 years.

Results: Mean age of the elderly and control groups was $78.2 \pm 3.1$ years and $68.9 \pm 2.7$ years, respectively $(p<0.001)$. Other clinical parameters were comparable between these two groups. The 1-, 3-, and 5-year graft survival rates in the elderly study group were $83.6 \%, 59.7 \%$, and $59.7 \%$, respectively, and those in the elderly control group were $79.4 \%, 68.1 \%$, and $59.6 \%$, respectively $(p=0.97)$. The overall 1-, 3-, and 5-year survival rates after donation from the elderly study group were $83.6 \%, 59.7 \%$, and $59.7 \%$, respectively, and those after donation from the control group were $79.3 \%, 72.1 \%$, and $64.1 \%$, respectively $(p=0.74)$. Regarding overall patient survival, univariate analysis identified pretransplant requirement for ventilator support $(p=0.021)$ and pretransplant renal replacement therapy $(p=0.025)$ as statistically significant risk factors; however, neither was significant on multivariate analysis.

Conclusions: The data suggest that organs from elderly donors do not worsen posttransplant outcomes; thus, advanced age should not be an exclusion criteria criterion. Indeed, using such donors could be the key to increasing the supply of liver grafts. 